\title{
El patrimonio gastronómico del municipio de Toluca: el caso del pulque y las pulquerías (1841-1920)
}

Edgar Rojas Rivas* y Felipe Carlos Viesca González*

Recepción: 13 de mayo de 2015

Aceptación: $\mathbf{3 0}$ de marzo de 2016

*Universidad Autónoma del Estado de México, México. Correos electrónicos: edgar.altermann@gmail.com;

Resumen. Se hace un esbozo de algunos elementos históricos del pulque y las pulquerías del municipio de Toluca en el periodo de 1841-1920, particularmente sobre la producción, comercialización y consumo. El método utilizado fue la microhistoria con información obtenida del Archivo Histórico del Municipio de Toluca y fuentes bibliohemerográficas. Los resultados muestran una producción significativa del pulque, la existencia de un gran número de establecimientos que lo expendían, la importancia del rol de la mujer en la comercialización y cómo el ferrocarril contribuyó a la distribución de la bebida. Se concluye que este patrimonio gastronómico fue importante para el municipio y que el consumo de la bebida era generalizado entre toda la población.

Palabras clave: pulque, pulquerías, microhistoria, patrimonio gastronómico.
The Gastronomic Heritage of the Municipality of Toluca: The Case of Pulque and Pulquerias (1841-1920)

Abstract. In this article we outline some historical elements of pulque and pulquerías in the municipality of Toluca in the period 18411920, particularly the production, marketing and consumption of the drink, are identified. The method used was microhistory with information obtained from the Historical Archive of the Municipality of Toluca and biblio-newspaper sources. The results show that there was a significant production of pulque in the municipality, there was a large number of establishments laid it out, the women were important in the marketing of the drink, and the railroad contributed to the distribution of the drink. We conclude that this gastronomic heritage was important for the municipality and the consumption of the beverage was widespread among the population.

Key words: pulque, pulquerías, microhistory, gastronomic heritage.

\section{Introducción}

El patrimonio, clasificado en natural y cultural, es definido como una serie de bienes que son heredados por generaciones anteriores y que constituyen una herencia hacia las futuras (Muñoz et al., 2004). Sobre el patrimonio cultural, García (1999) señala que debe servir para reconstruir la verosimilitud histórica de un pueblo. Bonfil (2004) lo define como un conjunto de símbolos, valores, actitudes, habilidades, conocimientos, significados, formas de comunicación y de organización social y bienes materiales que hacen posible la vida en una determinada sociedad. Por su parte, UNESCO (2016) se refiere a las tradiciones culinarias como una de las prácticas sociales en que se manifiesta el patrimonio cultural inmaterial. En 2010 la cocina tradicional mexicana fue inscrita en la Lista Representativa del Patrimonio Cultural Inmaterial de la Humanidad (unEsCO, 2010).

Sin embargo, el patrimonio gastronómico mexicano, y particularmente el del municipio de Toluca, ha sido un tema poco estudiado desde una perspectiva histórico- 
antropológica. Entre los escasos trabajos que lo abordan, sobresale la investigación de León (2002), quien estudia la riqueza gastronómica no sólo del municipio, sino también del valle de Toluca durante el periodo de 1750-1800. El libro de Sánchez (1976) menciona la vasta cantidad de productos de origen marranil que han distinguido a la municipalidad. La investigación de Romero et al. (2010) describe la formación del patrimonio gastronómico del valle de Toluca.

Trabajos más recientes de corte etnográfico, como los de González y Ovando (2010), Argüelles y Juárez (2010), Santillán (2011) y Rojas y Sánchez (2013) confirman la presencia de diversos alimentos patrimoniales entre los tolucences. Este artículo está enfocado en el pulque, una bebida casi extinta en Toluca. El objetivo fue identificar algunos elementos históricos del pulque y las pulquerías del municipio de Toluca y de esta manera destacar su importancia como patrimonio gastronómico. El trabajo abarca aspectos tanto de la producción, como de la comercialización y consumo de la bebida. El periodo de estudio fue de 1841 hasta 1920, ya que la segunda mitad del siglo XIX es considerada la época de mayor auge de la industria pulquera en el centro del país. Sin embargo, el surgimiento de la industria cervecera y las medidas tomadas en contra de la bebida durante las primeras décadas del siglo $\mathrm{xx}$ marcaron su declive.

La investigación realizada se centra en el municipio de Toluca, capital del Estado de México y uno de los municipios pertenecientes al valle de Toluca, que a su vez se encuentra en lo que hoy se denomina el Alto Lerma Mexiquense (Bastida, 2013). Aquí se usa "valle de Toluca" en el sentido que propone Albores (2013), el cual se empezó a utilizar desde el inicio de la colonia española para referirse al territorio que había ocupado en tiempos mesoamericanos del Posclásico la jurisdicción otomiana del Matlatzinco, a la jurisdicción novohispana de Hernán Cortés y posteriormente a distintas demarcaciones. Sobre todo desde el siglo xx, "valle de Toluca" se emplea para referirse a diferentes contenidos espaciales (Albores, 2013).

En los ámbitos académicos de la antropología e historia es común que se denomine como "valle de Toluca" a la porción del antiguo Matlatzinco situada en la subcuenca inicial del río Lerma, caracterizada por el Nevado de Toluca y el principal depósito acuático de toda la región, la Laguna de Lerma, el cual fue compartido por 20 municipios (Albores, 2013).

El método utilizado fue la microhistoria, propuesto por Luis González (1968). De acuerdo con González et al. (2005), lo que busca la microhistoria es ver como un pueblo ha transitado del pasado a su situación presente, y para ello se ocupa de los archivos que guardan información de los individuos comunes y corrientes, como los archivos municipales y parroquiales. Para la investigación fue indispensable consultar el Archivo Histórico del Municipio de Toluca (Анмт). Se revisaron documentos referentes a los negocios y personas vinculados a la producción y comercialización del pulque en el municipio de Toluca durante la época referida. Para poder recrear el contexto histórico socio-cultural del consumo de pulque en el municipio, se usó información de fuentes bibliohemerográficas de autores como Baranda y García (1987), García (1985), Garrido (1986), Loyola (1956), Salinas (1986), Venegas (1993) y Zárate (1901).

El documento se encuentra dividido en tres secciones: en la primera se da a conocer información sobre el contexto general del pulque y las pulquerías en México. En la segunda se menciona la importancia patrimonial de ambos en el municipio de Toluca, donde se muestran algunos datos sobre su producción y comercialización y se refieren aspectos socioculturales en relación con el consumo de la bebida. En la tercera sección se plantea una serie de conclusiones sobre el pulque y las pulquerías, la importancia histórica que tuvieron durante el siglo XIX y principios del xx y también se presenta una prospectiva del tema desarrollado.

\section{El pulque y las pulquerías en el Altiplano mexicano}

El pulque es una bebida que se obtiene a partir de la fermentación del aguamiel, líquido que se extrae del corazón de las plantas de algunas especies de agave pulquero como Agave americana, Agave atrovirens, Agave feroz, Agave mapisaga y Agave salmiana (Gómez et al., 2011; Alfaro et al., 2007). Asimismo, esta planta tiene muchos otros usos: delimitar los terrenos, evitar la erosión de los suelos, hospedero de los gusanos blanco y rojo, o las pencas son utilizadas para elaborar la barbacoa y de ellas también se obtiene la cutícula conocida como mixiote, empleada en varias preparaciones culinarias (Aguilar et al., 2014).

Hacer mención del pulque es hablar de un tema que a muchos disgusta y les genera repudio como resultado del desprestigio que se ha creado a la bebida (Piccato, 1997) y de algunas ideas difundidas respecto a su proceso de elaboración, por ejemplo que se le agrega excremento humano para fermentarlo. La propagación de esta última idea coincide con la llegada de la industria cervecera a nuestro país (Fernández y Deraga, 2007).

Aunado a lo anterior, las pulquerías han sido despreciadas y hostilizadas por varios grupos poblacionales, principalmente los habitantes de algunas zonas urbanas, ya que se cree que son lugares de embriaguez, poco higiénicos y donde los parroquianos se embrutecen, pierden la cabeza y después cometen actos inmorales que atentan contra la sociedad (Jiménez, 
2000), lo que ha generado que actualmente se encuentren casi extintas en muchas ciudades (Toxqui, 2008).

Contrastando dicha percepción, es necesario mencionar la importancia que tuvo el pulque durante la época prehispánica (Fournier y Mondragón, 2012), cuando solamente los ancianos podían consumirlo (Novo, 2010) y era utilizado con fines rituales (León, 2002). Sin embargo, con la llegada de los españoles perdió el carácter de bebida religiosa (Solange, 1989) y su consumo se incrementó entre la población indígena y española, lo cual dio como resultado la creación de la industria pulquera, que se desarrolló en el Altiplano Central de México, a mayor escala en algunas zonas de los estados de Hidalgo, Tlaxcala y el nororiente del Estado de México (Flores, 2004), aunque también estuvo presente en menor escala en otros lugares como San Luis Potosí, Querétaro y varios municipios del valle de Toluca.

El pulque del municipio de Toluca producido durante el siglo XVII era apreciado y de buena calidad. Casi todos los productores eran poderosos hacendados que se enriquecieron con la elaboración y comercialización de esta bebida, puesto que poseían grandes plantíos de maguey y muchas pulquerías en las principales ciudades del centro de México, incluyendo Toluca (León, 2002).

A mediados del siglo xvIII los indígenas de algunos grupos étnicos, como los otomíes y mazahuas, que comercializaban pulque en Toluca se resistieron a desarrollar dicha actividad en las pulquerías. Se dedicaban a esta actividad dentro de sus casas y salían a venderlo al centro y a las plazas de la ciudad solamente los días de mercado (que eran los viernes). El consumo de la bebida se fue generalizando a tal grado que a finales de la década de 1780 y principios de 1790, las ciudades de Puebla, México y Toluca eran los principales centros de consumo de pulque (León, 2002).

El pulque elaborado en Toluca fue adquiriendo buena fama en el transcurso del siglo XviII. Humboldt (1978) mencionaba que era el mejor y lo atribuía a las condiciones geográficas y el clima que prevalecían en esta zona. Rincón (1901) lo reafirmó de alguna manera al señalar que la verdadera zona de cultivo de maguey pulquero se encontraba entre los 2200 y 2700 msnm, y el municipio de Toluca se encuentra a una altitud de 2650 msnm (Biblioteca Enciclopédica del Estado de México, 1980).

A pesar de que la bebida no se producía en grandes volúmenes, en el municipio existía una gran cantidad de plantaciones de maguey pulquero; un ejemplo de lo anterior es la propiedad de la familia del español Tomás de Lexarazu, dueño de la Hacienda $3^{\text {a }}$ de S. Diego, la cual en 1791 contaba con 57883 magueyes en diferentes barrios y pueblos de Toluca (León, 2002).
Lo anterior muestra que no sólo los indígenas eran productores y consumidores de pulque, sino que también los españoles tenían un gran interés en ello (León, 2002). Ignacio Torres Adalid, un poderoso hacendado también conocido como el Rey del pulque (González, 1984), quien era descendiente de una vieja familia cuyo origen se remonta a la época Colonial (Ramírez, 2012), logró formar un verdadero emporio en el ramo del pulque y llegó a apropiarse de algunas pulquerías en el municipio de Toluca.

\section{El pulque y las pulquerías en el municipio de Toluca}

Como indica León (2002), la comercialización del pulque se realizaba de manera informal en el municipio. Probablemente su venta en establecimientos formales, conocidos también como cantinas de pulquería (Álvarez, 1997), se inició a finales de la década de 1830 y principios de 1840 en algunas plazuelas de la ciudad de Toluca, como la del Carmen, la Merced o el Coporito (Garrido, 1986) y en otros puntos de la ciudad. Para ese año existían 24 cantinas donde se vendían bebidas embriagantes, las principales eran el pulque y el aguardiente de caña. Algunas personas que tenían licencias para expender estas bebidas, contaban con otros negocios, como la señora Carmen Aspitia, dueña de una alacena en Los Portales.

En 1841 algunos habitantes de Toluca solicitaron al Ayuntamiento Municipal la apertura de cinco cantinas más en la plazuela del mercado de la ciudad. En ese mismo año se dieron licencias para la apertura de cinco cantinas mayores y tres menores; las primeras pagaban una pensión cuyo monto fluctuaba entre 1 y 6 pesos mensuales, mientras que las cantinas menores debían pagar una renta de doce reales mensuales (Анмт, Comercios, Caja 1, Expediente 8, 1839-1841); un real equivalía a 12.5 centavos, 1 peso era el equivalente a 8 reales (Toxqui, 2008). El costo del litro de pulque era de 4 centavos, de lo cual se deduce que sólo para cubrir la pensión mensual, las cantinas mayores debían registrar ventas de entre 25 y 1501 , mientras que las menores aproximadamente 37.51 .

Tradicionalmente los mercados han sido puntos importantes de venta de las bebidas tradicionales. En el mercado Riva Palacio, inaugurado el 16 de septiembre de 1851, existían 42 cantinas, entre las que se encontraban tiendas de abarrotes, vinaterías, empeños, bizcocherías, expendios de géneros del país (mantas y casimires), fondas, carnicerías y pulquerías (Baranda y García, 1987; Pedrero, 2001; Venegas, 1993). Se desconoce el número de cantinas de pulquería que existían en dicho mercado; sin embargo para esos años el Ayuntamiento recaudaba de ellas un total de 4260 pesos anuales (Baranda y García, 1987); gran parte de ese dinero debió haber tenido su 
origen en las pulquerías, las cuales pagaban rentas elevadas al ayuntamiento, lo cual sólo era posible si tenían éxito.

Las pulquerías no se encontraban únicamente en los mercados y en el centro de la ciudad, sino también en las periferias (Toner, 2011), plazas y pueblos del municipio. En la Plaza de los Mártires existía un buen número de estos negocios (Venegas, 1993); por ejemplo, el establecimiento del señor Pascual Betancourt, quien solicitó en 1852 que la cuota de 2 reales semanales que pagaba al Fondo Municipal por su negocio quedara reducida a la mitad (AHMT, Comercios, Caja 1, Expediente 17, 1859-1863). Probablemente los dueños de algunas pulquerías no podían cubrir todos los impuestos que se les asignaban y por eso solicitaban al municipio su condonación, ya que además tenían que pagar impuestos por abrir un expendio, refrendo de licencias y derecho de patente, entre otros rubros (Ramírez, 2012).

Para tener una idea de la demanda que había que satisfacer, según la Biblioteca Enciclopédica del Estado de México (1980), a mediados del siglo xIx el municipio contaba con una población de 26473 habitantes (11 836 hombres y 14637 mujeres); 8 años atrás tenía 24800 habitantes distribuidos de la siguiente manera: 8500 en la ciudad y sus barrios, 13500 en los pueblos del municipio y 2800 en las haciendas y ranchos (Salinas, 1986), estos últimos también contaban con su propia pulquería, a donde asistían los trabajadores al terminar su jornada laboral (Lagunas, 2011). En gran parte de los pueblos de la municipalidad existían personas que se dedicaban al cultivo del agave y producción de pulque (González, 2014).

Un trabajo encomendado por el Ministro de Fomento al Sr. D. Joaquín Noriega entre 1853 y 1854 (Biblioteca Enciclopédica del Estado de México, 1980), destaca que existían dos bebidas fermentadas de gran importancia en la dieta de los tolucenses: el pulque tlachique o desazonado (Hernández, 1979), obtenido a partir de magueyes de diferentes calidades y que tenía propiedades medicinales, pero era de menor calidad y más tóxico que el pulque fino (Godoy et al., 2003). La segunda es el sendecho, bebida elaborada a base de maíz amarillo, distintivo de las poblaciones otomíes y mazahuas (Moreno de los Arcos, 1975) y que era consumida en el noroeste de la ciudad de Toluca (Cruz y Ulloa, 1973).

Para 1859 se renovaron algunas disposiciones que dictaban que los dueños de las cantinas de pulquerías de Toluca debían colocar mostradores cerca de las puertas con el fin de que los consumidores no permanecieran más tiempo del necesario para consumir la bebida de pie o la pidieran para llevar. En estos negocios no se permitían reuniones, vendimias, música, juegos o diversiones; en caso de no cumplirlas, serían castigados con una multa de 1 a 5 pesos, como lo dictaba el artículo
27 de la ley establecida el 17 de febrero de 1857 (De Goribar, 1857) (Анмт, Comercios, Caja 1, Expediente 25, 1884). Estas disposiciones dictadas por el ayuntamiento estaban encaminadas a ejercer cierto control sobre el consumo de la bebida; a ellas se sumaba otra más dictada en 1856, según la cual las pulquerías debían cerrar a las 6:00 pm (Toner, 2011). De esta manera se manifestaba una preocupación de las autoridades por el exceso en el consumo y sus efectos en la población.

Durante la guerra civil entre liberales y conservadores en la década de 1860 , se dictaminaron y revocaron rápidamente numerosas disposiciones aplicables a las pulquerías de la ciudad de México (Toner, 2011; Toxqui, 2008). Para la década de 1870 la legislación del Distrito Federal definió que existirían dos tipos de pulquerías en la ciudad: las de venta interior y las de venta exterior. Las primeras serían como fondas y cantinas donde se permitiría la colocación de asientos para los clientes y también la venta de alimentos, mientras que en las segundas la venta del octli (pulque en náhuatl) sería para llevar o ser consumido en otros lugares (Toner, 2011).

Las fondas eran lugares donde se servía comida típica regional que solía ser acompañada con aguas frescas, cerveza o pulque, el cual no se expendía ahí, sino que era comprado en alguna pulquería cercana, ya que las fondas no tenían permiso para la venta de bebidas embriagantes, aunque podían estar abiertas durante toda la noche y muchas veces eran cantinas disfrazadas. Las personas que asistían a estos lugares eran de todas las clases sociales y buscaban degustar platillos auténticos de la comida mexicana, como enchiladas, chilatole de panza o huevos ahogados, de los cuales era característico su picor para aliviar la resaca (González, 1991).

El segundo tipo de pulquerías, las de venta exterior, son las de interés en este documento, ya que en el municipio de Toluca probablemente se haya aplicado la misma reglamentación que en el Distrito Federal. En el Archivo Histórico Municipal de Toluca se han encontrado numerosas solicitudes para la venta de pulque en la ciudad. Aunque las personas no contaban con las instalaciones necesarias para realizar esa actividad en sus hogares, sin duda era un negocio importante por la elevada demanda y por ello muchas personas solicitaban licencias para expender la bebida (Анмт, Comercios, Caja 1, Expediente 28, 1886).

En los zangarros o tendejones (tiendas pequeñas) (Gómez, 2001) también se expendía pulque y en la ciudad de Toluca había varios, como el de la señora Pascuala Tapia de Bermúdez, quien el 16 de abril de 1886 solicitó permiso al ayuntamiento para la venta en su negocio de uno o dos jarrones de tlachique. Durante el resto del año se siguieron otorgando permisos a más personas para la apertura de más pulquerías, como a Juan Reyes en la casa número 5 de la 
Plazuela del Carmen, Ramona Reynoso en la casa 10 de la calle de Santa Clara, Nazario Huerta para vender en una casilla de pulque en la Plazuela de la Merced, Eduardo Vázquez en la plaza del Tequesquite y Tomás Hernández, quien avisó a las autoridades que abrió una pulquería en la cantina del mercado número 32 con el nombre de La Bandera Roja (Анмт, Comercios, Caja 1, Expediente 28, 1886).

Algunos factores como el crecimiento económico del país, en particular del Altiplano Central, que era por excelencia la zona de cultivo de maguey pulquero (Lorenzo, 2007) y el aumento poblacional en las principales ciudades del centro del país, como la ciudad de México, Pachuca, Querétaro, Puebla, Tlaxcala y Toluca (que eran centros de distribución y consumo de pulque), propiciaron la apertura de numerosas pulquerías.

Para fines del siglo xix, Toluca casi había duplicado su población: según las Estadísticas sociales del Porfiriato 1877 1910 (Secretaría de Economía, 1956), en 1877 contaba con 12000 habitantes y para el año de 1895 tenía 23 150. Estos datos pueden servir como indicadores de la magnitud del incremento del consumo de pulque y del número de pulquerías que existían en municipio, puesto que de acuerdo con los "recibos de las cuotas que entran por las personas que expenden bebidas alcohólicas en sus respectivos comercios, lo mismo que los que expenden pulque en sus establecimientos" para el año de 1896 Toluca contaba con 81 pulquerías, la mayoría (51) concentradas en la ciudad del mismo nombre (Aнмт, Comercios, Caja 3, Expediente 2, 1896)

La cifra anterior es un número pequeño comparado con las mil pulquerías con que contaba la ciudad de México para el año 1900 (Ramírez, 2012) con una población de más de trece veces que la de Toluca. El medio rural municipal contaba con sus propios expendios de la bebida: San Mateo Oxtotitlán con siete, San Buenaventura, San Martín Toltepec y Santiago Tlacotepec con cuatro cada uno. El alto consumo de pulque en estos pueblos pudo haberse favorecido porque en ellos existía una gran cantidad de magueyes pulqueros.

Para 1896, no se han encontrado registros de pulquerías en Capultitlán, San Juan Tilapa, San Lorenzo Tepaltitlán, San Pablo Autopan, Santa Cruz Azcapotzaltongo, Santa Ana Tlapaltitlán, Santiago Tlaxomulco, Santa María Totoltepec, San Andrés Cuexcontitlán, San Cristóbal Huichochitlán, San Marcos Yachihuacaltepec y Santiago Miltepec. Esta situación se puede explicar porque algunos de ellos se encuentran al noroeste del municipio y colindan con los municipios de Almoloya de Juárez y Zinacantepec, probables proveedores de pulque de aquellos sitios. Otra posibilidad es que la producción en estos lugares era básicamente para autoconsumo y por lo tanto no se registraba su venta en alguna parte.
Los casos de los dos últimos municipios referidos son significativos, el primero por incluir en su territorio a la Hacienda La Gavia, la cual para 1893 tenía una extensión de 64000 hectáreas (Abasolo, 2006) y nunca se caracterizó por ser pulquera, pero en ella se producía el líquido, el cual se destinaba para consumo local y para los pueblos aledaños (Jarquín, 2004). A principios del siglo xx, la hacienda llegó a producir 3671751 de la bebida blanca, de donde se obtuvo una remuneración de 14687 pesos (Guzmán, 2010). Debe llamar la atención que a finales del siglo xix se empezó a registrar una importante producción de cebada que era destinada a la Compañía Cervecera Toluca y México s. A. (Abasolo, 2006); al parecer, aquí se replicó el mismo fenómeno que en otras regiones del país: superficies dedicadas al agave pulquero fueron sustituidas por la cebada, con el consiguiente desplazamiento del pulque por la cerveza.

Por otra parte, el municipio de Zinacantepec, colindante con Toluca, contaba con pueblos y haciendas con alta producción y consumo de pulque (Lagunas, 2011); en 1901 el ayuntamiento calculaba que en el municipio se producían 2090001 de pulque y existían 196000 magueyes cultivados (Almazán, 2011). Los lugares más importantes eran Santa María del Monte con 42000 magueyes, San Juan de las Huertas con 30000 y la Hacienda La Huerta con 39 000. Entre los tres acumularon una producción de 1130001.

Zinacantepec y Almoloya de Juárez no sólo abastecían de pulque a los pueblos de Toluca, sino que también de otros insumos como leña y nieve, que recogían de las zonas altas del volcán Xinantécatl para la elaboración y comercialización de helados (Almazán, 2011).

A fines del siglo xIx la producción de pulque en Toluca fue de 61320001 (Sánchez, 1896). La demarcación comprendía los municipios de Lerma, Metepec, Otzolotepec, Toluca, Zinacantepec y Almoloya de Juárez; los tres últimos eran los de mayor producción. Tal era la importancia del pulque tlachique en Toluca que su volumen de producción superaba a otros municipios como Zinacantepec.

Para 1902 en los municipios del valle de Toluca se reportó una producción de pulque tlachique de 11801921 superada casi tres veces por el pulque fino (Ramírez, 2012), de acuerdo con la "Noticia mensual del único producto que se elabora en la municipalidad de la producción de bebidas embriagantes". En el mismo año, la producción mensual de la bebida en Toluca se desglosaba de la siguiente manera: en enero había sido de 80050 1; para febrero, y de abril a noviembre, 800001 en cada mes; el total fue de 8000501 (Анмт, Comercios, Caja 4, Expediente 2, 1902). Asumiendo que en los meses de marzo y diciembre hubo una producción similar, la producción total para ese año se estima en 9600501. 
En las postrimerías del Porfiriato, el pulque se convirtió en la bebida más consumida en la República Mexicana, con el correspondiente incremento en el número de pulquerías, respecto a otros años. Entre 1907 y 1908, la Ciudad de México contaba con 1040 pulquerías (Ramírez, 2012). En Toluca el fenómeno también fue notorio. Según la "Lista que manifiesta el número de causantes que han sacado licencias y sus refrendos para expender bebidas embriagantes", entre 1899 y 1900 existían 120 pulquerías. En tres años se abrieron 69 pulquerías más respecto a 1896 (Анмт, Comercios, Caja 3, Expediente 9, 1899-1900).

En la ciudad existían varias personas que concentraban la venta de la bebida. El caso más notorio es el de Tirso Parra, quien tenía diez pulquerías ubicadas principalmente en lugares estratégicos como las avenidas Independencia, Riva Palacio, Lerdo, y sitios como El Callejón del Garabato, Huitzila, Ferrocarriles Nacionales de México y el Mercado Riva Palacio. Tirso Parra promocionaba los expendios de pulque fino que poseía en otros lugares como El Oro, Maravatío, Acámbaro y Morelia (Zárate, 1901).

Respecto al rol que desempeñaron las mujeres en este proceso, en la "Lista que manifiesta el número de causantes que han sacado licencias y sus refrendos para expender bebidas embriagantes" (Анмт, Comercios, Caja 3, Expediente 9, 1899-1900), aparecen los nombres de 27 mujeres que vendían pulque en la ciudad. Kanter (1992) explica que desde finales del siglo XVIII y principios del XIX, algunas mujeres del valle de Toluca, específicamente de Tenango del Valle, se dedicaban a la raspa de maguey y a la venta de pulque, actividades que constituían el sostén familiar, ya que probablemente eran viudas o madres solteras. Oficialmente las mujeres dejaron de comercializar pulque hasta 1919, cuando Carranza decretó que los expendios de bebidas alcohólicas no podían ser administrados por ellas (Barbosa, 2004).

Por otra parte, el crecimiento industrial y demográfico que experimentó el país durante la segunda mitad del siglo xIx y principios del xx hizo que muchos varones se incorporaran al ámbito industrial y abandonaran las actividades agrícolas. En Toluca las principales industrias que surgieron fueron la cervecera, a través de la Compañía Cervecera Toluca y México S. A., la textilera, con fábricas como La Industria Nacional, y de conservas alimenticias y empacadoras de productos derivados del cerdo. Otras industrias importantes en el estado eran la de hilados de algodón en Tlalnepantla y Amecameca, de lana en Tlalmanalco y la vidriera en Texcoco y San Rafael (Montes de Oca, 1998).

En 1907 la Administración de Rentas Municipales de Toluca solicitó una lista con el número y clase de establecimientos mercantiles y talleres de artes y oficios inscritos en los registros públicos de la ciudad, y en ella se registran 121 expendios de pulque, uno más respecto a 1899-1900 (AHMT, Comercios, Caja 5, Expediente 6, 1907).

El gran auge de la industria pulquera en esa época era evidente, ejemplo de ello son las diez licencias que en 1909 el ayuntamiento le concedió el señor Javier Piña y Aguayo para abrir expendios de pulque, entre los cuales figuraban El Baco Divino, en la calle de Independencia número 45; El Árbol, en la calle 5 de Mayo, y El Triunfo, en la avenida Juárez.

Personajes pertenecientes a la aristocracia pulquera, como Ignacio Torres Adalid, tenían pulquerías en Toluca, pero no se cuenta con el dato preciso del número de ellas. De lo que se tiene conocimiento es que El rey del pulque, como se conocía a Torres, las tenía arrendadas al Dr. Isidro Izquierdo ( $2^{\circ}$ regidor del municipio) (AнмT, Comercios, Caja 8, Expediente 1, 1909). Ramírez (2012) menciona que entre los intereses de la familia Torres Adalid estaba lograr transportar el pulque a Toluca, Tenango y Temascaltepc; algo que pudo haber logrado, ya que si era dueño de varias pulquerías en la ciudad de Toluca, probablemente también envió el líquido a estas zonas a través del ferrocarril.

El ferrocarril jugó un papel importante en la expansión de la industria pulquera en el valle; entre 1902 y 1918 las líneas Toluca-Tenango-Atlatlahuca y Toluca-San Juan de las Huertas registraban a la bebida como el segundo producto más transportado, junto con el maíz, paja, cebada, harina, frutas y legumbres, heno, semillas, tabaco y trigo representados por 29098.3 ton, sólo superados por los productos forestales con 89218.6 ton (Flores, 2012).

Pero, ¿de dónde se obtenían tales cantidades de pulque? Se desconoce la cantidad de magueyes que existía en el municipio y dónde se encontraban las plantaciones; sin embargo, a partir de los casos de las haciendas de Almoloya y Zinacantepec se puede inferir que las haciendas de Toluca debieron tener importantes extensiones de maguey pulquero y ser de las principales productoras de la bebida. Por supuesto, también había muchos pequeños productores, como el Sr. Nicolás Mercado, quien tenía sembradas algunas plantas en el barrio de San Sebastián (Aнмт, Tierras, Caja 5, Expediente 8, 1912).

El trabajo de Loyola (1956) puede ayudar a deducir el número de magueyes que existían en Toluca. Se parte del supuesto de que para 1930 cada maguey producía 1301 de pulque en un lapso de tres meses, es decir, un promedio de 1.451 diariamente. Tomando en cuenta que en 1902 se produjeron 9600501 de pulque en Toluca, se puede inferir que hubo una producción media de 26301 de pulque al día; ahora bien, si cada maguey producía 1.451 aproximadamente, se tiene que por lo menos había 1814 magueyes produciendo 
pulque durante tres meses y sobre una base de un año. En total se estimaría esa cifra en 7256 magueyes.

En el municipio de Toluca había cinco haciendas, cada una con más de mil hectáreas, las cuales producían principalmente cebada, haba, trigo y maíz (Abasolo, 2006). De este último cereal se llegaron a producir $603628 \mathrm{~kg}$ en Canaleja y $186120 \mathrm{~kg}$ en Santín (Aнмт Agricultura, Ganadería y Fomento, Caja 2, Expediente 17, 1872-1919). Probablemente los magueyes estaban intercalados entre los cultivos de cereales; de ser así, Loyola (1956) menciona que los agaves eran plantados a una distancia de 5 a $10 \mathrm{~m}$, dejando espacio para los otros cultivos, y concluye que había aproximadamente doscientas plantas por hectárea.

De esta manera, se puede inferir que había alrededor de treinta y seis hectáreas cultivadas de maguey pulquero distribuidas en los ranchos, haciendas, terrenos de particulares u otros puntos del municipio, como en los cerros del Calvario o del Toloche (Salinas, 1986). Ahora bien, eso no significaba que solamente existieran 7256 magueyes pulqueros. Para 1930, se reporta que por cada planta explotada, existían otras treinta y siete que no producían pulque debido a que no estaban en edad para ello; con base en lo anterior, se puede argumentar que a principios del siglo xx, la población aproximada de magueyes en Toluca era de 268472.

El pulque formaba parte importante de la dieta alimenticia de los tolucenses Escamilla (2001) y Garrido (1986) refiere que era una bebida de los pobres. Sin embargo, dado el gran número de establecimientos que había y otras evidencias, se puede aseverar que se consumió de manera generalizada entre la población, al menos durante un periodo de tiempo. Incluso, durante el siglo xix los alumnos del Instituto Científico Literario solían tomar pulque. Badia (2004: 23) proporciona una descripción de las comidas que realizaban los alumnos en el refectorio del Instituto:

Se desayuna (4:00 a 6:00 am): café con pan o té de monte (limón, cedrón, naranjo, canela); el almuerzo (10:00 a 12:00 pm): huevo con frijoles y salsas, tortillas o pan de sal, arroz rojo con chícharos; la comida (1:00 a 3:00 pm): papas hervidas, charales, carpas cocidas, elotes con cebollas y chile verde, maíz con calabacitas y frijoles negros, habas con tortillas y era tradicional el pulque, agua o té de hojas o hierbas; y la merienda (5:00 a 7:00 pm): tortillas, frijoles, quelites hervidos y refritos con cebollitas y salsita, corazones de María acompañados de atole con piloncillo, café, agua y cemitas.

El consumo de pulque también se realizaba en las fiestas de la ciudad. En esas ocasiones había personas que solicitaban licencias temporales para la venta de la bebida. Por ejemplo, en 1909: el señor Juan Bernal, para la fiesta del barrio de Santa Clara en agosto; el señor Carlos Jiménez para los días 3 y 4 de octubre durante la fiesta de San Miguel que se realizó en la calzada de La Retama; el señor E. González para el 22 de septiembre durante las fiestas de la Virgen de la Merced en el Jardín de los Hombre Ilustres de la ciudad (Aнмт, Comercios, Caja 8, Expediente 1, 1909).

\section{Conclusiones}

Los documentos encontrados en el Archivo Histórico del Municipio de Toluca dan cuenta de la importancia patrimonial del pulque en Toluca y de las numerosas pulquerías que existían en el municipio. Contrariamente a lo que refieren algunos autores como Garrido (1986), que mencionan que el pulque era una bebida para una clase social baja, se ha mostrado que durante el periodo estudiado, su consumo era generalizado entre los tolucenses.

Se ofrece un esbozo sobre la producción, comercialización y consumo del pulque y las pulquerías del municipio de Toluca en el periodo estudiado y se ha evidenciado la importancia que tuvo esta industria. El valle de Toluca era considerado pulquero, a pesar de que la cantidad y calidad del pulque producido en éste no se comparaban con los Llanos de Apan. Existían numerosos establecimientos que expendían pulque en la ciudad y pueblos. Bajo ciertas condiciones, la mujer llegó a desempeñar un rol importante en la comercialización de la bebida.

De la misma manera que en el resto del centro del país, en el municipio de Toluca el ferrocarril contribuyó a la circulación de la bebida. Personajes de la aristocracia pulquera, como Ignacio Torres Adalid o Tirso Parra, tenían numerosas pulquerías en la ciudad y muy probablemente lograron transportar la preciada bebida desde los Llanos de Apan hasta Toluca, a través del ferrocarril.

\section{Prospectiva}

Actualmente el patrimonio gastronómico constituido por el maguey, pulque y pulquerías de Toluca se encuentra en vías de desaparecer, ya que esta bebida ha sido desplazada por otras como el tequila, mezcal, brandy o cerveza, siendo esta última la de mayor preferencia entre la población (INPRFM, 2011). Otros elementos que han impactado significativa y negativamente en el consumo de pulque en el municipio son los discursos que denigran a la bebida señalando que la fermentación del aguamiel se realiza con excremento humano.

$\mathrm{El}$ abandono del maguey es notorio debido a la falta de interés de los campesinos y de las nuevas generaciones para continuar con su cultivo, originado por factores 
como la disminución de la demanda del pulque, el bajo precio al que se oferta y el largo periodo de espera hasta que la planta empieza a producir el aguamiel (la materia prima para elaborar el pulque), mientras tanto queda expuesta al robo de la cutícula, como lo refieren Aguilar et al. (2014). Aunado a lo anterior, cada vez son menos las personas que producen y comercializan la bebida, ya que continúa estigmatizada como una bebida desagradable y exclusiva de pobres, además de contar con pocos canales de comercialización.

Pese a todo, la planta multiusos del maguey y la otrora bebida de los dioses se resisten a morir: en Toluca existen todavía pequeñas zonas de cultivo ubicadas en diversos sitios. La venta del líquido se sigue realizando en los tianguis de los pueblos, fiestas, mercados y pulquerías por productores provenientes de Zinacantepec, Temoaya, Xonacatlán y Jiquipilco. En algunos grupos sociales, principalmente entre los jóvenes y académicos, pareciera que la bebida está de moda, tal vez por curiosidad y como un intento de revalorizarla.

Más allá de etiquetarlas como lugares perjudiciales para la sociedad, las escasas pulquerías de Toluca que aún sobreviven se podrían reconocer como lugares con un valor patrimonial, ya que guardan una riqueza cultural e histórica.
Lo escrito por los hermanos Fabila (1951) a mediados del siglo pasado continúa vigente: si el pulque, siendo una bebida con buenas propiedades alimenticias y alto contenido nutrimental (Sierra et al., 2015), está desprestigiado y es motivo de menosprecio social es porque se le ha perseguido, extorsionado (aún al día de hoy) y relegado a la inmundicia. Es indispensable que se supriman esos complejos, que la bebida se proteja y produzca higiénicamente para facilitarla a la gente que la quiera consumir, y así sea tan sana y tan recomendada como el vino o la cerveza.

De esa manera se preservaría uno de los alimentos emblemáticos del Estado de México, se extendería el cultivo de la planta en todo su territorio, se disfrutaría de sus beneficios ambientales y se generarían mayores ingresos para más productores.

Tal vez no esté lejano el día en que el pulque vuelva a ser la bebida nacional, sustituyendo al tequila y al mezcal, por factores como su característica de líquido natural (sin la incorporación de otras sustancias) y bajo contenido alcohólico, la sobreexplotación que se está realizando del tequila y mezcal, la demanda del mercado de nuevas experiencias sensoriales, y la buena percepción que tiene entre la población local, visitantes y turistas, al menos en Toluca (Rojas et al., 2016), como probablemente sucede en muchas regiones del país

Archivos consultados

Ramo Agricultura, Ganadería y Fomento

Agricultura, Ganadería y Fomento (1872-

1919), Caja 2, Expediente 17, Анмт

(Archivo Histórico del Municipio de Toluca): Toluca

Ramo Comercios

Comercios (1909), Caja 8, Expediente 1, Aнmт (Archivo Histórico del Municipio de Toluca): Toluca.

Comercios (1907), Caja 5, Expediente 6, (Archivo Histórico del Municipio de Toluca): Toluca.

Abasolo, V. (2006). Entre el cielo y la tierra: raíces, un pueblo de la alta montaña del Estado de México (tesis de doctorado). México: Universidad Iberoamericana.

Aguilar, B., Enríquez, J., Rodríguez, G., Gra-
Comercios (1902), Caja 4, Expediente 2 (Archivo Histórico del Municipio de Toluca): Toluca.

Comercios (1899-1900), Caja 3, Expediente

9 (Archivo Histórico del Municipio de Toluca): Toluca

Comercios (1896), Caja 3, Expediente 2 (Archivo Histórico del Municipio de Toluca): Toluca.

Comercios (1886), Caja 1, Expediente 28, (Archivo Histórico del Municipio de Toluca): Toluca.

Comercios (1884), Caja 1, Expediente 25 (Archivo Histórico del Municipio de Toluca): Toluca

Comercios (1859-1863), Caja 1, Expediente 17 (Archivo Histórico del Municipio de Toluca): Toluca.

Comercios (1839-1841), Caja 1, Expediente 8 (Archivo Histórico del Municipio de Toluca): Toluca.

Ramo Tierras

Tierras (1912), Caja 5, Expediente 8 (Archivo Histórico del Municipio de Toluca): Toluca.

Referencias

nados, D. y Martínez, B. (2014). El estado actual de Agave salmiana y A. mapisaga del Valle de México. Revista Mexicana de Agroecosistemas, 1(2), 106-120.

Albores, B. (2013). Zanbatha. Etapas históri- cas del Valle de Toluca, en M. Bastida, B. Albores y G. Patrick (coords.), Zanbatha. Valle de la Luna (pp. 5-58). México: Universidad Autónoma MetropolitanaUnidad Lerma. 
Alfaro, G., Legaria, J. y Rodríguez, J. (2007).

Diversidad genética en poblaciones de agaves pulqueros (Agave spp.) del nororiente del Estado de México. Revista Fitotecnia Mexicana, 30(1), 1-12.

Almazán, M. (2011). Usos, perspectivas y conflictos por los recursos forestales en los pueblos de la montaña (Nevado de Toluca) durante el porfiriato, 1876-1911 (tesis de maestría). México: CIEsas.

Álvarez, J. (1997). Las cantinas: donde la palabra se bumedece. México: Editorial edamex.

Argüelles, M. y Juárez, R. (2010). Análisis de los sistemas de producción y comercialización de tortilla de maíz en la ciudad de Toluca (tesis de licenciatura). México: UAem.

Badia, G. (2004). Breve reseña bistórica del Instituto Literario de la cindad de Toluca hasta la conformación de la Universidad Autónoma del Estado de México (tesis de maestría). México: Universidad Iberoamericana.

Baranda, M. y García, L. (1987). Estado de México: textos de su historia. México: Instituto Mora y Gobierno del Estado de México.

Barbosa, M. (2004). Controlar y resistir. Consumo de pulque en la Ciudad de México, 1900-1920. Segundo Congreso Nacional de Historia Económica. Simposio: Las bebidas alcohólicas, siglos XVIII-Xx: producción, consumo y fiscalidad. El Colegio de México.

Bastida, M. (2013). El Alto Lerma Mexiquense, en M. Bastida, B. Albores y G. Patrick G. (coords.), Zanbatha. Valle de la Luna (pp. 59-74). México: Universidad Autónoma Metropolitana-Unidad Lerma.

Biblioteca Enciclopédica del Estado de México (1980). Estadística del Departamento de México. Formada por la Comisión nombrada por el Ministerio de Fomento, y presidida por el Sr. D. Joaquin Noriega; de septiembre 1853 en que comenzó sus trabajos, a febrero de 1854 en que los concluyó. México: Biblioteca Enciclopédica del Estado de México.
Bonfil, B. (2004). Pensar nuestra cultura. Nuestro patrimonio cultural: un laberinto de significados. Diálogos en la acción, primera etapa (117-134).

Cruz, S. y Ulloa, M. (1973). Alimentos fermentados de maíz consumidos en México y otros países Latinoamericanos. Revista de la Sociedad Mexicana de la Historia Natural, $34,423-457$.

De Goribar, J. (1857). Arreglo de policía para la ciudad de Toluca, en 17 de febrero de 1857. España: Biblioteca Nacional de España.

Escamilla, A. (2001). Los paseos dominicales en Toluca durante el Porfiriato. México: UAem.

Fabila, A. y Fabila, G. (1951). México. Ensayo socioeconómico del Estado. Volumen I. México: Talleres Gráficos de la Nación.

Fernández, R. y Deraga, D. (2007). The use of waste Products in the fermentation of alcoholic beverages, en J. MacClancy J. Henry y H. Macbeth (coord.), Consuming the inedible: Neglected dimensions of food choice (pp. 215-222). usA: Berghahn Books.

Flores, J. (2004). Breve historia de la comida mexicana. México: DeBolsillo.

Flores, N. (2012). Los constructores del ferrocarril Toluca-San Juan de las Huertas, 1883 1906. Contribuciones desde Coatepec, 23, 95-113.

Fournier, P. y Mondragón, L. (2012). Las bebidas mexicanas. Pulque, mezcal y tesgüino. Revista Arqueología Mexicana, 19(114), 52-59.

García, M. (1985). Toluca en el Porfiriato. México: Gobierno del Estado de México. García, N. (1999). Los usos sociales del Patrimonio Cultural, en A. Encarnación (ed.), Patrimonio Etnológico. Nuevas perspectivas de estudio (pp. 16-33). España: Consejería de Cultura de la Junta de Andalucía.

Garrido, I. (1986). La ciudad de Toluca. México: Gobierno del Estado de México.

Godoy, Q., Herrara, T. y Ulloa, M. (2003). Bebidas alcohólicas no destiladas indígenas de México. México: unam.
Gómez, C., Díaz, C., Villaruel, A., Torres, M., Añorve, J., Rangel, E., Cerna, J., Vigueras, J. y Castro, J. (2011). Behavior of Salmonella Typhimurium, Staphylococcus aureus, Listeria monocytogenes, and Shigella flexneri and Shigella sonnei during production of pulque, a traditional Mexican beverage. Journal of Food Protection, 74(4), 580-587.

Gómez, G. (2001). Diccionario breve de mexicanismos. México: Academia Mexicana, Fondo de Cultura Económica.

González, J. (1991). Las cantinas y las fondas en las postrimerías del porfiriato (19001910). La Palabra y el Hombre, 79, 252-256. González, L. (1968). Pueblo en vilo: microbistoria de San José de Gracia. México: El Colegio de México.

González, L. (1984). La ronda de las generaciones. México: SEP.

González, L. y Ovando, P. (2010). Alimentosy bebidas tradicionales que se ofrecen en las cantinas de la ciudad de Toluca y el comportamiento de sus actores sociales (tesis de licenciatura). México: UAEm.

González, L., Martínez, C. y Aguirre, C. (2005). Mesa redonda: microhistoria mexicana, microhistoria italiana e historia regional. Relaciones, 26(101), 193-224.

González, M. (2014). El tribunal de vagos en Toluca 1845-1853. México: Instituto Mexiquense de Cultura.

Guzmán, X. (2010). México en la hacienda de La Gavia. La hacienda de La Gavia en México, 1774-1950 (tesis de doctorado). México: unam.

Hernández, J. (1979). La renta del pulque en la Nueva España, 1663-1810. España: Consejo Superior de Investigaciones Científicas.

Humboldt, A. (1978). Ensayo politico sobre el reino de la Nueva España. México: Porrúa. INPRFM (Instituto Nacional de Psiquiatría Ramón de la Fuente Muñiz) (2011). Encuesta Nacional de Adicciones: Reporte de Alcohol. México: INPRFM. 
Jarquín, M. (2004). La Gavia: historia de la transformación de una hacienda. Economia, Sociedad y Territorio, 4(16), 759-764.

Jiménez, A. (2000). Lugares de gozo, retozo, abogo y deshago en la Ciudad de México. Cantinas, pulquerias, hoteles de rato, sitios de prostitución y cárceles. México: Océano.

Kanter, D. (1992). Viudas y vecinos, milpas y magueyes. El impacto del auge de la población en el Valle de Toluca: el caso de Tenango del Valle en el siglo xvin. Estudios Demográficos y Urbanos, 7, 19-33.

Lagunas, H. (2011). Vida laboral y cotidiana en las haciendas de Zinacantepec, siglos XIx y xx. La Colmena, 70, 83-95.

León, M. (2002). Distinción alimentaria de Toluca: el delicioso valle y los tiempos de escasez, 1750-1800. México: Porrúa.

Lorenzo, A. (2007). Las haciendas pulqueras en México. Colección de Estudios de Posgrado. Programa de Posgrado en Arquitectura. México: UnAm.

Loyola, E. (1956). La industria del pulque. México: Banco de México.

Montes de Oca, E. (1998). Bibliografía comentada. El Estado de México durante el porfiriato: (1876-1910). Documentos de Investigación, 13, 1-23.

Moreno de los Arcos, R. (1975). Una lista de bebidas alcohólicas del siglo xviII. Notas Antropológicas, 1, 170-179.

Muñoz, M., Sanhueza, R., Seguel, L. y López, M. (2004). La participación social y protección del patrimonio. Urbano, 7(10), 19-23.

Novo, S. (2010). Cocina mexicana. Historia gastronómica de la ciudad de México. México: Porrúa.

Pedrero, G. (2001). Los trabajadores en Toluca durante el siglo xix. El sector Económico, en J. Aranda y G. Zamudio (coord.), Valle de Toluca: devenir social y cultural (pp. 33-51). México: UAEM.

Piccato, P. (1997). El discurso sobre la criminalidad y el alcoholismo hacia el fin del
Porfiriato, en R. Pérez (coord.), Hábitos, normas y escándalo. Prensa, criminalidad y drogas durante el Porfiriato tardio (pp.77-144). México: ciesas, Plaza y Valdés Editores.

Ramírez, M. (2012). El Rey del Pulque. Ignacio Torres Adalid y la industria pulquera. México: UNAM.

Rincón, P. (1901). El maguey. Memoria presentada por el General Pedro Rincón Gallardo, presidente de la Comisión Agrícola Mexicana en el Congreso Internacional de Agricultura que funcionó en la ciudad de Forth Worth, Texas del 6 al 14 de diciembre de 1898. Imprenta particular de la S. Agrícola Mexicana.

Rojas, E. y Sánchez, D. (2013). Las tortas: patrimonio gastronómico de Toluca y su supervivencia ante los establecimientos de comida rápida (tesis de licenciatura). México: Uaem.

Rojas, E., Viesca, F., Espeitx, E. y Quintero, B. (2016). El maguey, el pulque y las pulquerías de Toluca, Estado de México, ¿patrimonio gastronómico turístico? Pasos (14) 5, 1199-1215.

Romero, A., Viesca, F. y Hernández, M. (2010). Formación del patrimonio gastronómico del Valle de Toluca, México. CIENCIA ergo-sum, 17(3), 239-252.

Salinas, M. (1986). Datos para la bistoria de Toluca. México: Gobierno del Estado de México. Sánchez, A. (1976). Toluca del chorizo. Apuntes gastronómicos. Serie de Arte popular y Folklore. México: Gobierno del Estado de México.

Sánchez, T. (1896). El alcoholismo en la República Mexicana. Discurso pronunciado en la sesión solemne que celebran las sociedades científicas y literarias de la nación el día 5 de junio de 1896. México: Imprenta del Sagrado Corazón de Jesús.

Santillán, Á. (2011). Caracterización morfológica y fisicoquimica del chorizo verde que se comercializa en la ciudad de Toluca: estudio preliminar (tesis de licenciatura). México: uaem.
Secretaría de Economía (1956). Estadísticas sociales del Porfiriato 1877-1910. México: Secretaría de Economía.

Sierra, E., Marcial, J., García, B., Mendoza, J., Gómez, S. y Enríquez, S. (2015). Molecular identification of yeast of the pulque by PCRDGGE, a traditional mexican beverage. International Journal of ResearchGranthaalayah, 3(3), 1-15.

Solange, A. (1989). Bebidas alcohólicas y sociedad colonial en México: un intento de interpretación. Revista Mexicana de Sociología, 51(2), 349-359.

Toner, D. (2011). Everything in its right place? Drinking places and social spaces in Mexico City, C. 1780-1900. Social History of Alcohol and Drugs, 25(1), 26-48.

Toxqui, M. (2008). El recreo de los amigos. Mexico City's pulquerias during the liberal republic (1856-1911) (tesis de doctorado). USA: University of Arizona.

UNESCO (United Nations Educational, Scientific and Cultural Organization) (2016). Social practices, rituals and festive events. Consultado el 23 de marzo de 2016. Disponible en http://www.unesco.org/ culture/ich/en/social-practices-ritualsand-00055

unesco (United Nations Educational, Scientific and Cultural Organization) (2010). La cocina tradicional mexicana, cultura comunitaria, ancestral y viva-El paradigma de Michoacán. Consultado el 23 de marzo de 2016. Disponible en http:// www.unesco.org/culture/ich/es/RL/ la-cocina-tradicional-mexicana-culturacomunitaria-ancestral-y-viva-el-paradigma-de-michoacan-00400

Venegas, A. (1993). Guia del viajero en Toluca. México: Instituto Mexiquense de Cultura. Zárate, F. (1901). Toluca antigua y moderna: álbum del Estado de México. La administración del General Villada. España: Biblioteca Nacional de España. 